\title{
An NMDA Receptor Signaling Complex with Protein Phosphatase 2A
}

\author{
Shing Fai Chan and Nikolaus J. Sucher \\ Department of Biology and Biotechnology Research Institute, The Hong Kong University of Science and Technology, \\ Clear Water Bay, Kowloon, Hong Kong Special Administrative Region, China
}

\begin{abstract}
Regulation of protein phosphatase 2A (PP2A) activity and NMDA receptor (NMDAR) phosphorylation state contribute to the modulation of synaptic plasticity, yet these two mechanisms have not been functionally linked. The NMDAR subunit NR3A is equipped with a unique carboxyl domain that is different from other NMDAR subunits. We hypothesized that the NR3A C-terminal intracellular domain might serve as synaptic anchor for the phosphatase in the developing CNS. A cDNA library was screened by the yeast two-hybrid method using the NR3A carboxyl domain as the bait. The catalytic subunit of the serine-threonine PP2A was found to be associated with the NR3A carboxyl domain. Immunoprecipitation studies indicated that the NR3A subunit formed a stable complex with PP2A in the rat brain in vivo. Association of PP2A with NMDARs led to an increase in the phosphatase activity of PP2A and the de-
\end{abstract}

phosphorylation of serine 897 of the NMDAR subunit NR1. Stimulation of NMDARs led to the dissociation of PP2A from the complex and the reduction of PP2A activity. A peptide corresponding to the PP2A-NR3A binding domain functioned as a negative regulator of PP2A activity. These data suggest that NMDARs are allosteric modulators of PP2A, which in turn controls their phosphorylation state. The data delineate a mechanistic model of the dynamic regulation of a PP2ANMDAR signaling complex, mediated by the interaction of NR3A and PP2A, and suggest a novel NMDAR-mediated signaling mechanism in addition to the traditional ionotropic functions of NMDARs.

Key words: protein phosphatase 2A; NMDA; phosphorylation; synapse; neuron; brain; rat
Signaling through protein phosphorylation is of importance for the normal development and function of the nervous system (Walaas and Greengard, 1991; Roche et al., 1994; Levitan, 1999; Swope et al., 1999). The phosphorylation state of neuronal proteins is tightly balanced and controlled in vivo by the opposing activities of protein kinases and phosphatases. The dynamic nature of protein phosphorylation serves to maintain the efficacy and specificity of intracellular signals, which participate in the modulation of phosphorylation status, and substrate specificity of signal transduction proteins (Faux and Scott, 1996; Smart, 1997). Accumulating evidence demonstrates that both the temporal and spatial extent of protein kinases and phosphatases are regulated by an intricate system of protein-protein interactions (Ziff, 1997; Fraser and Scott, 1999).

Protein phosphatase 2A (PP2A) is one of the major serinethreonine phosphatases that exists as a multisubunit enzyme complex and is expressed at high levels in the CNS (Strack et al., 1998). The enzyme complex consists of a $36 \mathrm{kDa}$ catalytic subunit (PP2A-C) and a $65 \mathrm{kDa}$ structural subunit (PP2A-A) forming a core enzyme, which then associates with a variable regulatory

\footnotetext{
Received June 19, 2001; revised Aug. 7, 2001; accepted Aug. 8, 2001.

Part of this work was supported by Research Grants Council Grants HKUST6100/ 98M and HKUST6135/99M. We thank Dr. G. Walter at the University of California, San Diego, for the monoclonal antibody 6F9, Dr. B. A. Hemmings at the Friedrich Miescher-Institute for the PR65 and PR55 $\alpha$ antibodies, Dr. J. S. Trimmer at the State University of New York in Stony Brook for the anti-NR3A antibody, Dr. R. L. Huganir at the Howard Hughes Medical Institute, The John Hopkins University School of Medicine, for the anti-phospho-NR1 antibodies, Dr. D. Krainc for the cDNA library, and Dr. M. Carles for critical reading of this manuscript.

Correspondence should be addressed to Dr. Nikolaus J. Sucher, Department of Biology, Hong Kong University of Science and Technology, Clear Water Bay, Kowloon, Hong Kong SAR, China. E-mail: sucher@ust.hk.

Copyright (C) 2001 Society for Neuroscience $\quad 0270-6474 / 01 / 217985-08 \$ 15.00 / 0$
}

subunit (PP2A-B) to constitute the heterotrimeric PP2A holoenzyme (Mumby and Walter, 1993). PP2A plays key roles in many fundamental cellular processes, including signal transduction (Mumby, 1995) and gene expression (Schonthal, 1995). There are intriguing functional similarities between PP2A and NMDA receptors (NMDARs). Both contribute to the regulation of neural functions such as synaptic transmission and plasticity. For example, previous studies have shown that one form of synaptic plasticity, long-term depression (LTD) is thought to be dependent on the change of the phosphorylation state of glutamate receptors in general (Lee et al., 1998, 2000) and the activity of phosphatases, possibly PP1 and PP2A in particular (Mulkey et al., 1993, 1994; Thiels et al., 1998). However, the functional relationship between PP2A and NMDARs and its molecular determinants has not been defined (Wang et al., 1994).

NMDARs are heteromultimeric complexes that are comprised of at least two types of subunits, the principal subunit NR1 and the modulatory subunit NR2A-D (Moriyoshi et al., 1991; Meguro et al., 1992; Monyer et al., 1992). Both types of subunits have carboxyl intracellular domains (Kutsuwada et al., 1992) that interact with associated proteins that regulate NMDAR function (Sheng and Pak, 1999). Recently, an additional developmentally regulated NMDAR subunit NR3A has been identified (formerly called $\chi$-1 or NMDAR-L) (Ciabarra et al., 1995; Sucher et al., 1995). This receptor subunit has been shown to attenuate NMDA-mediated currents when co-expressed with NR1 and NR2 subunits in Xenopus oocytes (Ciabarra et al., 1995; Sucher et al., 1995). Cortical neurons from NR3A "knock-out" mice showed enhanced NMDA responses and increased dendritic spine density (Das et al., 1998). NR3A is equipped with a unique carboxyl domain that is different from other NMDAR subunits. 
We undertook the present study to investigate whether the NR3A $\mathrm{C}$-terminal intracellular domain might serve as a target for associated proteins with signaling function in the developing CNS.

\section{MATERIALS AND METHODS}

Yeast two-hybrid screen. The NR3A cDNA encoding the full-length intracellular C terminus (NR3Ac, Gly ${ }^{1}-\mathrm{Ser}^{165}$ ) was amplified by PCR. The resulting PCR products were purified using the QIAquick PCR purification kit (Qiagen, Hilden, Germany) and subsequently subcloned into the expression vector pEG202 in-frame with a LexA DNA-binding domain. This expression construct, together with a URA reporter plasmid and a human fetal brain cDNA library in plasmid pJG4-5, was transformed into yeast strain Saccharomyces cerevisiae EGY48 by the lithium acetate method to screen for NR3A-associated proteins. The transformants were selected on the basis of the formation of (1) blue colonies on selective plates lacking uracil, histidine (-His), and tryptophan, and containing 5-bromo-4-chloro-3-indolyl- $\beta$-D-galactosidase, and (2) LEU gene expression from a chromosomal lexAop-LEU reporter on plates lacking leucine (-Leu). Expression of the lacZ reporter gene was determined by measuring $\beta$-galactosidase activity using 2 -nitrophenyl- $\beta$ D-galactopyranoside as substrate as described previously (Rose et al., 1990). A series of NR3A C-terminal deletions were constructed (NR3Ac-1, Gly ${ }^{1}-\mathrm{Phe}^{37}$; NR3Ac-2, Gly ${ }^{1}-\mathrm{Gln}^{20}$; NR3Ac-3, Tyr ${ }^{21}-\mathrm{Phe}^{37}$; NR3Ac-4, Leu ${ }^{8}$-His $^{30}$; NR3Ac-5, Val ${ }^{38}$-Ser ${ }^{165}$; NR3Ac-6, Leu ${ }^{132}$ $\mathrm{Ser}^{165}$ ), transformed into yeast strain Saccharomyces cerevisiae EGY48, and assayed for $\beta$-galactosidase activity. The sequences of the constructs were verified by DNA sequencing using the AutoRead Sequencing Kit (Amersham Pharmacia Biotech, Arlington Heights, IL).

Construction of an NR3A without the intracellular carboxyl-domain by site-directed mutagenesis. Site-directed mutagenesis was performed as described previously (Ho et al., 1989). A pair of primers (5'-CC ATC CTG ACC ACC ATT TGA GAA CAC ATA GTG CAC AG-3' and 5'-CT GTG CAC TAT GTG TTC TCA AAT GGT GGT CAG GAT GG-3') encoding the desired mutations was used to change the first codon of the NR3A intracellular carboxyl domain into a stop codon that generated an NR3A protein lacking the intracellular carboxyl-domain (NR3A $\Delta-\mathrm{c}$ ). Mutations were confirmed by DNA sequencing.

Preparation of synaptic plasma membranes and postsynaptic densities. Sucrose gradient extraction method was used to prepare synaptic plasma membranes (SPMs) and postsynaptic densities (PSDs) as previously described (Rogers et al., 1991). Protein concentration of SPM and PSD was determined by bicinchoninic acid protein assay (Sigma, St. Louis, MO).

Preparation of cerebrocortical neurons. Cerebrocortical neurons were prepared from the cerebral cortex of mice at embryonic day 16. Cerebral cortices freed of meninges were incubated in $10 \mathrm{ml}$ of $0.25 \%$ trypsin in HBSS without calcium and magnesium (Life Technologies, Gaithersburg, MD) for $5 \mathrm{~min}$ at $37^{\circ} \mathrm{C}$. Then, the cell suspension was sieved through a $70 \mathrm{~mm}$ sterile Mesh nylon filter (Spectrum Medical Industries, New Delhi, India), diluted in warm DMEM containing $10 \%$ fetal bovine serum and $2 \mu \mathrm{M}$ L-glutamine (Life Technologies), and plated on culture dishes coated with $12.5 \mu \mathrm{g} / \mathrm{ml}$ poly-D-lysine (Sigma). The cells were exposed to $40 \mu \mathrm{M}$ cytosine arabinoside (Sigma) after day 3 and incubated for $24 \mathrm{hr}$ to inhibit astrocytic growth. At day 7 after in vitro seeding, cultures were harvested, and the resulting cell pellets were resuspended in RIPA buffer and homogenized in a Potter-Elvehjem homogenizer. The homogenates were centrifuged at $20,800 \times g$ for $10 \mathrm{~min}$ at $4^{\circ} \mathrm{C}$ to remove the nuclei and mitochondria. The supernatant was subsequently used for immunoprecipitation and Western blotting analysis.

Transfection of human embryonic kidney (HEK) 293 cells and stimulation of NMDARs in transfected HEK 293 cells and cerebrocortical neurons. For transient expression of heteromeric NMDARs, including NR1, NR2B, and NR3A, HEK 293 cells were transiently transfected using calcium phosphate precipitation. A total of $28 \mu \mathrm{g}$ of cDNA were transfected per $100 \mathrm{~mm}$ tissue culture plate. The NR1, NR2B, and NR3A cDNAs were transfected in a 1:2:4 ratio. To prevent NMDAR-mediated cell death of transfected cells, $20 \mu \mathrm{M}$ 7-chlorokynurenate was added to the culture media. Forty-eight hours after transfection, cells were harvested and homogenized in RIPA buffer (150 mM sodium chloride, $1 \%$ NP-40, $0.5 \%$ deoxycholic acid, $0.1 \%$ SDS, $50 \mathrm{~mm}$ Tris- $\mathrm{HCl}, \mathrm{pH} 7.5,1 \mathrm{~mm}$ EDTA, pH 8, 1 mM EGTA, pH 8, and $20 \mu \mathrm{M}$ 7-chlorokynurenate). Activation of NMDARs in cerebrocortical neurons and transfected HEK 293 cells expressing heteromeric NMDARs was induced by adding 200 $\mu \mathrm{M}$ NMDA, $10 \mu \mathrm{M}$ glycine, and $2.5 \mathrm{~mm}$ calcium in nominally $\mathrm{Mg}^{2+}$-free
Hank's solution at $37^{\circ} \mathrm{C}$ for $5 \mathrm{~min}$. Cell extracts were prepared immediately after the exposure to NMDA.

Assay of endogenous PP2A activity. Endogenous PP2A activity was determined by the serine-threonine phosphatase assay (Promega, Madison, WI). Tissue samples from rat brain, cell lysates of transfected HEK 293 cells, and cerebrocortical neurons were homogenized in RIPA buffer containing a freshly prepared protease inhibitor mixture of $1 \mathrm{~mm}$ phenylmethylsulfonyl fluoride (PMSF), $20 \mu \mathrm{g} / \mathrm{ml}$ benzamidine, $10 \mu \mathrm{M}$ leupeptin, $1 \mu \mathrm{M}$ pepstatin, and $20 \mu \mathrm{M}$ 7-chlorokynurenate. Protein concentration of homogenates was determined by bicinchoninic acid protein assay. A total of $5 \mu \mathrm{g}$ of homogenate was used for assay. A PP2A inhibitor, $1 \mathrm{~nm}$ okadaic acid (OA) (Life Technologies Inc.), was added and incubated at $30^{\circ} \mathrm{C}$ for $30 \mathrm{~min}$.

Peptide synthesis. Synthetic peptide (SP1) was purified by HPLC (Genemed Synthesis, San Francisco, CA). The amino acid sequence of the synthetic peptide was: GEHIVHRLLLPRIKNKSKLQYWLHTSQRFHRALNTSF.

Immunoprecipitation, gel electrophoresis, and immunoblotting. Protein samples from rat brain membrane fractions, $600 \mu \mathrm{g}$ of SPM and PSD, and cell lysates of transfected HEK 293 cells and cerebrocortical neurons were used for immunoprecipitation. Primary antibody $(10 \mu \mathrm{g} / \mathrm{ml})$ was added and incubated for $2 \mathrm{hr}$ at $4^{\circ} \mathrm{C}$, followed by $2 \mathrm{hr}$ incubation with GammaBind Plus Sepharose (1:1 slurry in RIPA buffer). Immunoprecipitates were eluted from GammaBind Plus Sepharose in sample loading buffer (126 mm Tris-HCl, pH 6.8, 20\% glycerol, 4\% SDS, 1 м urea, 300 $\mathrm{mm}$ dithiothreitol, $1 \mathrm{~mm}$ PMSF, and $0.005 \%$ bromophenol blue) at $100^{\circ} \mathrm{C}$ for $7 \mathrm{~min}$. Immunoprecipitated samples were analyzed by SDS-PAGE and immunoblotting after electro-transfer of proteins to polyvinylidene difluoride membranes. Antibodies used for immunoblotting included anti-NR3A, provided by J. S. Trimmer (State University of New York, Stony Brook, NY); anti-NR1 (PharMingen, San Diego, CA); antiphospho-NR1 (Upstate Biotechnology, Lake Placid, NY); 6F9 against PP2A core enzyme and holoenzyme, provided by G. Walter (University of California, San Diego, CA); PR65 and PR55 $\alpha$ antibodies, provided by B. A. Hemmings (Friedrich Miescher-Institut, Basel, Switzerland); and anti-PP2A catalytic subunit (PharMingen). Immunoblots were developed by enhanced chemiluminescence (ECL; Amersham Pharmacia Biotech) and visualized on Hyperfilm (Amersham Pharmacia Biotech). The films were scanned into Adobe Photoshop with a Microtek optical scanner and stored as gray-scale images in TIFF file format. The intensity of labeling was quantified by measuring the gray value of the pixels contained in a rectangular area matching the bands of interest. The mean intensity of labeling obtained for each antibody band was calculated by subtracting the background value (measured at the site of the corresponding antibody band in the IgG lane). For the developmental profile of rat brain, the value obtained at postnatal day 0 (P0) for both NR1 and NR3A (as illustrated in Fig. 5D) was selected as a reference point, and all other values were normalized to the reference point and expressed as percentage.

\section{RESULTS}

\section{Identification of PP2A as an NR3A interacting protein and characterization of the PP2A-NR3A interaction domain}

A human fetal brain cDNA library was screened by the yeast two-hybrid method using the full-length carboxyl intracellular domain of the NR3A subunit as the "bait." Approximately $1,000,000$ clones were screened, and initially 36 clones were identified as interactors. After DNA sequence analysis and database screening using the National Center for Biotechnology Information Basic Local Alignment Search Tool, one clone was identified as the $\beta$-isoform of the catalytic subunit of the serine-threonine PP2A. Transformation of yeast cells confirmed that this clone interacted with NR3A and gave the strongest signal among the other clones in a $\beta$-galactosidase assay. These results indicated that the carboxyl intracellular domain of the NR3A subunit appeared to contain a PP2A binding site. To further define the nature of interaction between NR3A and PP2A, various bait fragments containing different amino acid cassettes were constructed and assayed for their binding activities to PP2A (Fig. 1). The strength of the interaction between the $\mathrm{N}$-terminal 37 amino 


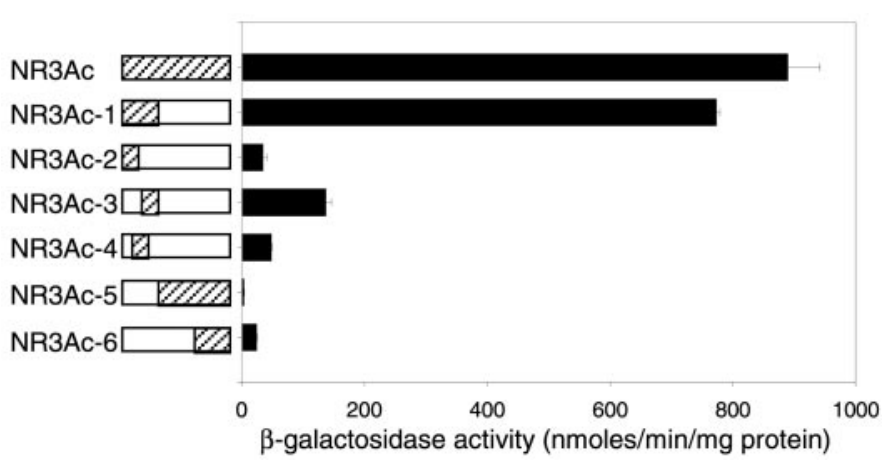

Figure 1. Interaction of NR3A with PP2A. The entire carboxyl intracellular domain of NR3A (NR3Ac: Gly $^{1}-\mathrm{Ser}^{165}$ ) and various deletion constructs (NR3Ac-1-NR3Ac-6) were used in a yeast two-hybrid assay. Hatched areas correspond to the constructs used in the assay; open boxes indicate the deletions that were made from the carboxyl intracellular domain. Corresponding $\beta$-galactosidase activities of each construct on interaction with PP2A are summarized in the histogram. $\beta$-galactosidase activities are given in nanomoles per minute per milligram of protein \pm SEM.

acid cassette of the NR3A intracellular domain (NR3Ac-1) and PP2A was similar to that of the full-length carboxyl intracellular domain (NR3Ac). Other bait fragments, NR3Ac-2-4, containing truncated regions of NR3Ac-1, and NR3Ac-5 and - 6 lacking the entire region of NR3Ac-1, were found to bind weakly to PP2A (Fig. 1). These results suggested that the N-terminal 37 amino acid cassette of the NR3A intracellular domain was necessary and sufficient for binding PP2A.

\section{Association of PP2A with NR3A mediated through the carboxyl intracellular domain of NR3A}

To investigate whether the association of PP2A with NR3A could be detected in mammalian cells, HEK 293 cells were transiently transfected with full-length NR3A cDNA that was cloned previously into the mammalian expression vector pBK-CMV. Cell extracts from transfected HEK 293 cells were then used for immunoprecipitation. Anti-PP2A monoclonal antibody 6F9, which was shown previously to recognize and immunoprecipitate specifically the PP2A core enzyme and holoenzyme (Kremmer et al., 1997), was used for these experiments. The 6F9 antibody co-immunoprecipitated PP2A with NR3A, whereas a control antibody (anti-rat IgG) did not (Fig. $2 A$ ). The expression of PP2A subunits was detected in lysates from HEK 293 cells transiently transfected with NR3A (Fig. 2B) by immunoblotting. These results showed that the PP2A formed a complex with NR3A in mammalian cells. Next, we examined whether the carboxyl intracellular domain of NR3A was essential for interacting with PP2A in mammalian cells. A mutant of NR3A lacking the carboxyl intracellular domain (NR3A $\Delta$-c) was constructed by sitedirected mutagenesis (Fig. $2 C$ ). This deletion mutant was transfected into HEK 293 cells, and the cell extracts were subsequently used for immunoprecipitation. No co-immunoprecipitation (coIP) between NR3A $\Delta$-c and PP2A was detected (Fig. 2D). The lack of association between PP2A and NR3A $\Delta$-c indicated that the carboxyl intracellular domain of NR3A was necessary for the PP2A-NR3A interaction in mammalian cells.

\section{Co-immunoprecipitation of PP2A with NR3A in rat brain membrane fractions}

Similarly to the NMDAR subunit NR1, both NR3A and PP2A were detected in fractions enriched in SPMs and PSDs (Das et al.,
1998; Kennedy, 2000) (Fig. 2E), a structure thought to be important in clustering and anchoring of glutamate receptors. To determine whether PP2A directly interacted with NR3A in the brain, immunoprecipitation was performed in SPM and PSD fractions from rat brain. NR3A protein was detected in immunoprecipitates from both SPM and PSD fractions when anti-PP2A monoclonal antibody $6 \mathrm{~F} 9$ was used but not in immunoprecipitates in which a nonspecific anti-rat $\mathrm{IgG}$ antibody was used as control (Fig. $2 F$ ). These results provided direct evidence that PP2A was associated with NR3A in the brain consistent with the results in HEK 293 cells.

\section{NMDAR-activity-dependent association of PP2A with NR3A in transfected HEK 293 cells and neurons}

After establishing a physical link between PP2A and NR3A, it was of interest to identify specific signals governing the formation of the PP2A-NR3A complex. Therefore, we conducted experiments to investigate whether the NMDAR channel activity could regulate the association of the $\mathrm{PP} 2 \mathrm{~A}$ with NR3A in mammalian cells. Transfection of HEK 293 cells with vectors for NMDAR subunits NR1 + NR2B + NR3A was performed and the cell extracts were used for immunoprecipitation. Triple combination of transfection was performed because expression of NR3A by itself did not lead to the formation of NMDAR channels in Xenopus oocytes (Ciabarra et al., 1995; Sucher et al., 1995) and in HEK 293 cells (Pérez-Otaño et al., 2001), but expression of NR3A with NR1 and NR2 subunits gave rise to NMDAR-gated channels with unique properties. These included low $\mathrm{Ca}^{2+}$ permeability and attenuation of NMDA-mediated currents in those expression systems (Pérez-Otaño et al., 2001). NR3A protein was detected in anti-PP2A (6F9) immunoprecipitates in the absence of NMDAR stimulation by NMDA (Fig. $3 A$ ). In contrast, when NMDARs were activated by NMDA (200 $\mu \mathrm{M}$ NMDA, $10 \mu \mathrm{M}$ glycine, and $2.5 \mathrm{mM} \mathrm{Ca}^{2+}$ in nominally $\mathrm{Mg}^{2+}$-free Hank's solution), PP2A-NR3A co-IP was not detected (Fig. $3 A$ ). At the same time, no significant change was observed in immunoprecipitates of the NMDAR subunit NR1 or co-IP of that subunit with NR3A (Fig. 3A). Similar results were obtained in cerebrocortical neurons (Fig. $3 B$ ). The expression of PP2A subunits was detected in lysates from cerebrocortical neurons (Fig. $3 C$ ). These results support the notion that NMDAR channel activity could elicit "on" and "off" signals to selectively regulate the association of PP2A with NMDAR in HEK 293 cells and neurons.

\section{Selective dephosphorylation of NR1 and allosteric activation of endogenous PP2A activity by the formation of an PP2A-NR3A complex}

NMDAR subunit NR1 is expressed ubiquitously in the brain and is the principal subunit for the formation of recombinant NMDARs in neurons (Moriyoshi et al., 1991; Meguro et al., 1992; Monyer et al., 1992). To delineate the functional consequences of the association of PP2A with NMDARs, we investigated whether the PP2A-NMDAR complex could influence the phosphorylation state of the essential NMDAR subunit NR1. Transfection of HEK 293 cells with combinations of NR1 + $\mathrm{NR} 2 \mathrm{~B}+\mathrm{NR} 3 \mathrm{~A}$ and NR1 + NR2B + NR3A $\Delta$-c was performed, and cell extracts were used for immunoprecipitation with antiNR1 antibody. The phosphorylation state of NR1 was determined by immunoblotting with anti-phospho-NR1 antibody (specific for phosphorylation on Ser 897 residue on NR1) (Tingley et al., 1997). Phospho-NR1 was virtually undetectable in HEK 293 cells expressing NR1 + NR2B + NR3A. In contrast, a significant level of anti-phopsho-NR1 was observed in HEK 293 cells ex- 
A

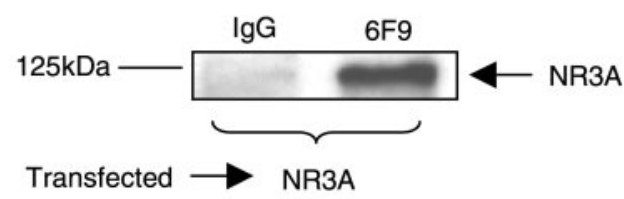

B

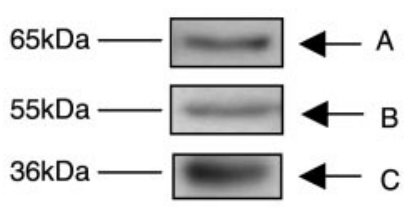

C
D

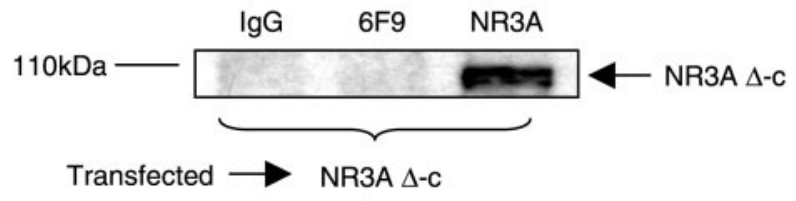

E

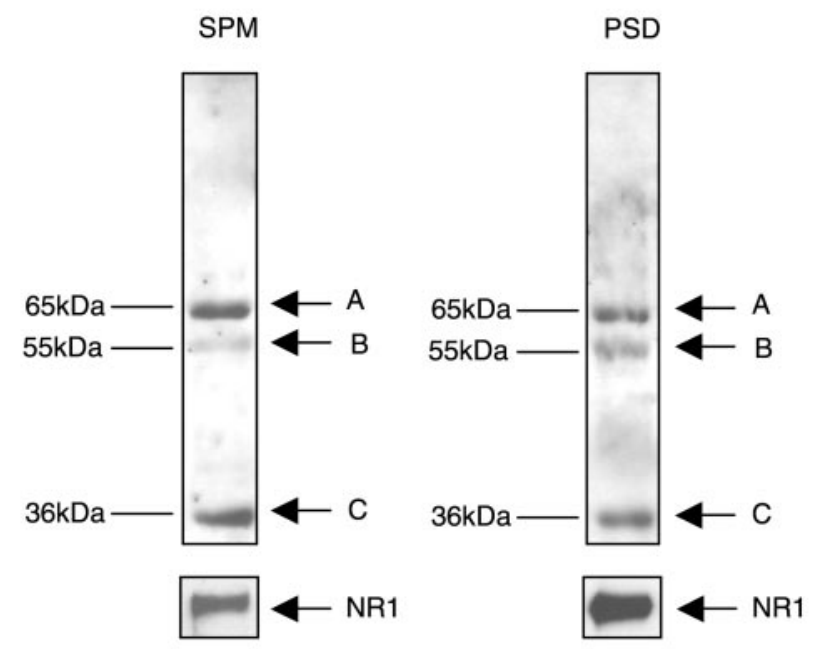

F

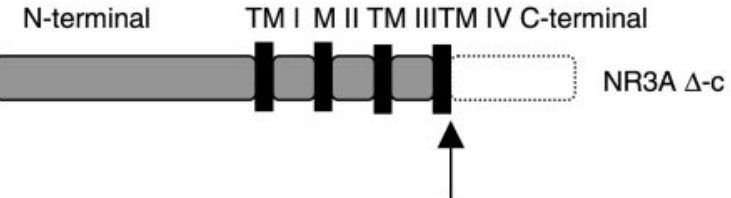

Stop codon
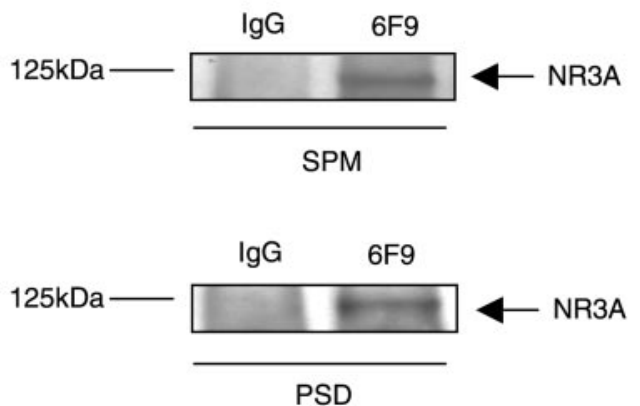

Figure 2. PP2A-NR3A interaction mediated through the carboxyl intracellular domain of NR3A. A, Lysates from HEK 293 cells transiently transfected with NR3A were immunoprecipitated with control $\operatorname{IgG}$ antibody (anti-rat $\mathrm{IgG}$ ) and anti-PP2A monoclonal antibody $6 \mathrm{~F} 9$. Western blots were probed with monoclonal antibody anti-NR3A. Data are representative of six experiments showing similar results. B, Lysates from HEK 293 cells transiently transfected with NR3A were separated by SDS-PAGE, and the expression of PP2A was detected by probing with antibody PR65 (recognizing the A-subunit), antibody PR55 $\alpha$ (recognizing the B-subunit), and antibody anti-PP2Ac (recognizing the C-subunit). C, Schematic drawing of NR3A $\Delta$-c. The part of the carboxyl intracellular domain of NR3A that was deleted is indicated by the dotted line. TM I-IV designates transmembrane regions I-IV. $M$ II designates membrane loop M II. D, Lysates from cells transfected with NR3A $\Delta$-c were immunoprecipitated with control IgG antibody (anti-rat IgG) or monoclonal antibody $6 \mathrm{~F} 9$. Western blots were probed with monoclonal antibody anti-NR3A to detect the expression of NR3A $\Delta$-c. $E$, Coimmunoprecipitation of PP2A with NR3A in rat brain membrane fractions. Lysates from rat brain membrane fractions were separated by SDS-PAGE, and the expression of PP2A and NR1 was detected by probing with antibody PR65 (recognizing the A-subunit), antibody PR55 $\alpha$ (recognizing the B-subunit), antibody anti-PP2Ac (recognizing the C-subunit), and anti-NR1. F, Immunoprecipitations from synaptic plasma membrane (SPM) and postsynaptic density $(P S D)$ fractions from rat brain were performed using control IgG antibody and monoclonal antibody 6F9, which was used to immunoprecipitate PP2A (core enzyme and/or holoenzyme). Western blots were probed with monoclonal antibody anti-NR3A.

pressing NR1 + NR2B + NR3A $\Delta$-c or when NR1 + NR2B + NR3A-expressing cells were stimulated by the addition of NMDA (Fig. 4A). These results provided evidence that Ser 897 of the NR1 subunit was a direct or indirect in vivo target for PP2A-mediated dephosphorylation of NMDARs. Furthermore, the difference between the phosphorylation level of NMDARs in HEK 293 cells expressing NR1 + NR2B + NR3A and NR1 +
$\mathrm{NR} 2 \mathrm{~B}+\mathrm{NR} 3 \mathrm{~A} \Delta-\mathrm{c}$ or NR1 + NR2B + NR3A (stimulated by the addition of NMDA) indicated that the phosphorylation state of NMDARs was specifically influenced by the formation of a PP2A-NMDAR complex.

Next, we asked whether the enzymatic activity of PP2A was influenced by the formation of the PP2A-NMDAR complex. HEK 293 cells were transfected with a triple combination of NR1 


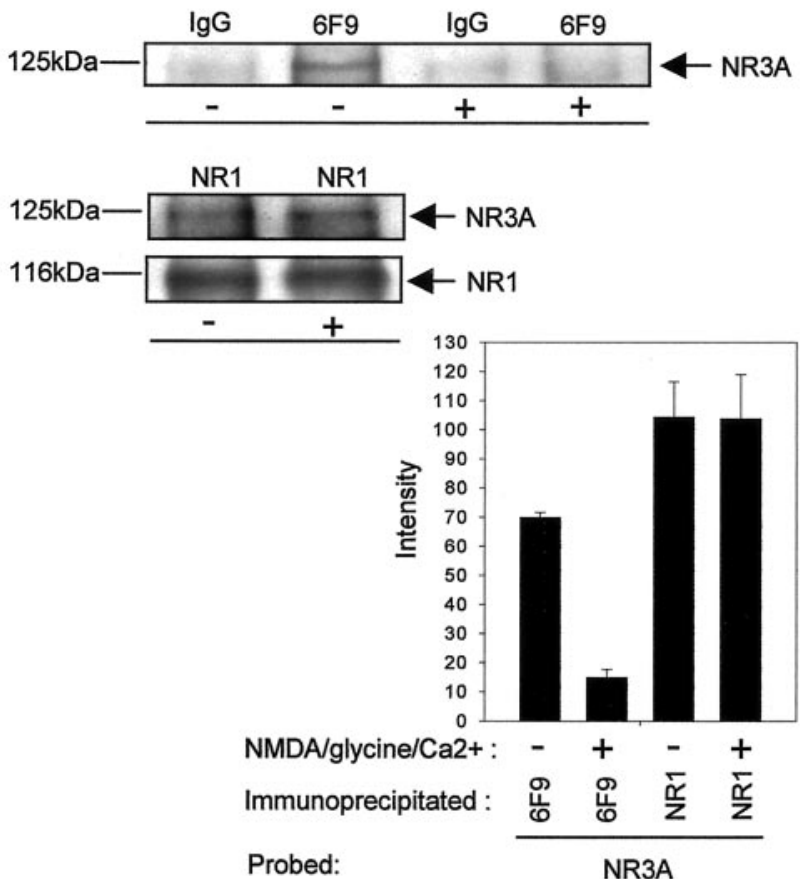

B

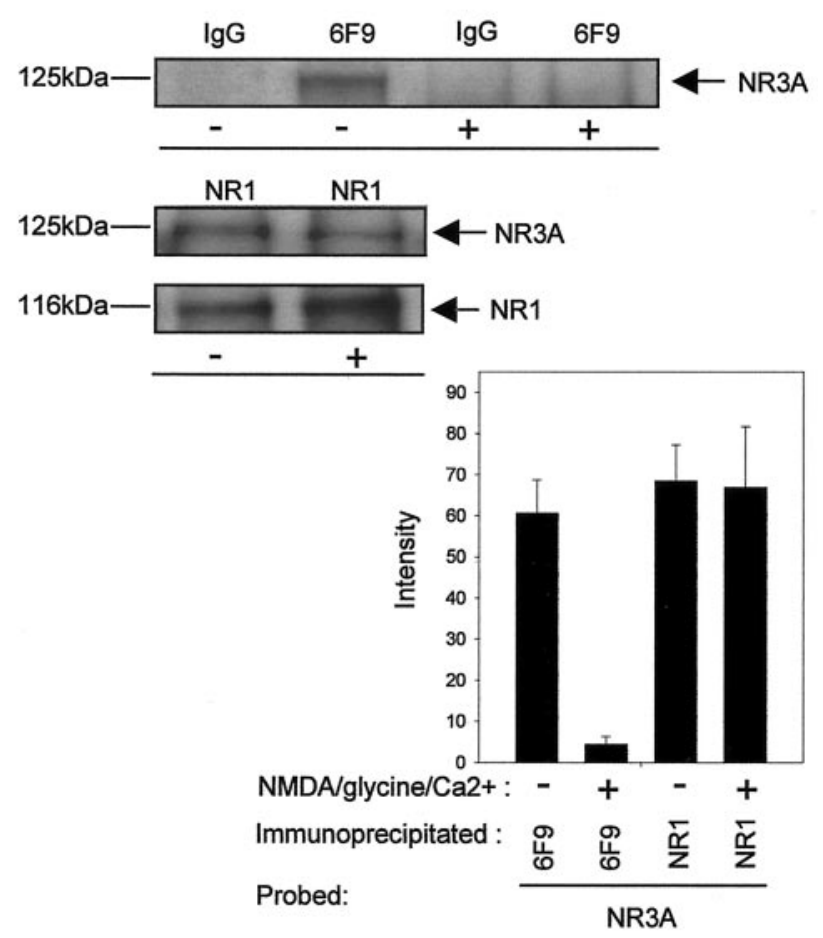

C

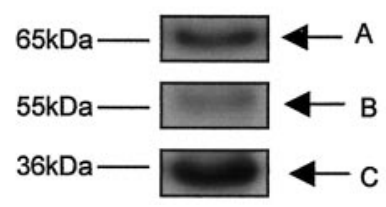

Figure 3. NMDAR activity-dependent association of PP2A with NR3A in transfected HEK 293 cells and neurons. A, HEK 293 cells expressing heteromeric NMDARs consisting of NR1 + NR2B + NR3A were incubated in the absence or presence of NMDA (200 $\mu \mathrm{M}$ NMDA, $10 \mu \mathrm{M}$ glycine, and $2.5 \mathrm{mM} \mathrm{Ca}^{2+}$ in nominally $\mathrm{Mg}^{2+}$-free Hank's solution). Immunoprecipitations were performed using control $\mathrm{IgG}$ antibody, mono-
$+\mathrm{NR} 2 \mathrm{~B}+\mathrm{NR} 3 \mathrm{~A}$ or NR1 + NR2B + NR3A $\Delta$-c or NR3A alone. Solubilized extracts from these cells were used to assay endogenous PP2A activity. PP2A activity was found to be increased more than eightfold in cells transfected with NR1 + $\mathrm{NR} 2 \mathrm{~B}+\mathrm{NR} 3 \mathrm{~A}$ compared with the control (8.6 $\pm 0.5 ; n=6 ; p<$ 0.01 ) ( Fig. $4 B$ ). Such an increase in PP2A activity was observed neither in cells transfected with NR1 + NR2B + NR3A that were treated with NMDA nor in cells transfected with the triple combination consisting of NR1 + NR2B + NR3A $\Delta$-c or NR3A alone (Fig. 4B). These data suggested that the formation of a complex between NMDARs and PP2A, mediated by the interaction of the carboxyl intracellular domain of NR3A and PP2A, activated PP2A. On the other hand, stimulation of NMDARs by the agonist NMDA in the presence of the co-agonist glycine and the absence of magnesium led to the dissociation of PP2A from the complex resulting in the attenuation of PP2A activity. In addition, co-expression of NMDAR subunits NR1, NR2B, and NR3A that would lead to the formation of NMDAR channels was required for the regulation of $\mathrm{PP} 2 \mathrm{~A}$ activity.

\section{Blockade of endogenous PP2A activity by a synthetic peptide in transfected HEK 293 cells, cultured neurons, and rat brain membrane fractions}

A peptide (SP1) corresponding to the PP2A-NR3A binding domain was synthesized. To test its effect on the PP2A-NR3A interaction and the resulting activation of PP2A activity, solubilized extract fractions from HEK 293 cells expressing NR1 + NR2B + NR3A were incubated with SP1, and the effect of this peptide on endogenous PP2A activity was determined. A significant reduction of endogenous PP2A activity was observed in protein extracts from HEK 293 cells expressing NR1 + NR2B + NR3A that were treated with SP1 $(5 \mu \mathrm{M})$ compared with control $(76.1 \pm 1.3 \% ; n=3 ; p<0.001)$ (Fig. $5 A)$. The inhibitory effect of SP1 was similar to the blocking effect of the PP2A inhibitor, OA (Fig. 5A). A dose-response relationship curve for the effect of SP1 on PP2A activity was constructed, and the data were fit to the Hill equation (Fig. 5B). SP1 inhibited PP2A activity with an inhibitory concentration at half maximum $\left(\mathrm{IC}_{50}\right)$ of $1.5 \mu \mathrm{M}$. To test whether SP1 could inhibit PP2A activity in cultured neurons and the brain, the peptide was added to the solubilized extract fractions from cerebrocortical neurons in culture and rat brain at different stages of development (postnatal days $0,4,8,12$, and 16, and adult). Similar to its effect in HEK 293 cells, SP1 inhibited

\section{$\longleftarrow$}

clonal antibody 6F9, and NR1. Top, Western blots probed with monoclonal antibody anti-N R3A and anti-NR1. Bottom, left, Intensity (mean gray level $\pm \mathrm{SD}$ ) of NR3A labeling in the presence (co-IP with $6 \mathrm{F9}, 14.9 \pm 2.8$; co-IP with NR1, $103.7 \pm 15.3$ ) and absence (co-IP with 6F9, $69.2 \pm 2.3$ co-IP with NR1, $104.3 \pm 12.1$ ) of NMDAR stimulation (co-IP with 6F9, $p<0.00005$; co-IP with NR1, $p<0.5 ; n=3$ separate experiments). $B$, Cells from cerebrocortical neurons were incubated in the absence or presence of NMDA. The cell lysates were subsequently immunoprecipitated with control IgG antibody or monoclonal antibody $6 \mathrm{~F} 9$ or NR1. Top, Western blots probed with monoclonal antibody anti-NR3A and antiNR1. Bottom, left, Intensity (mean gray level $\pm \mathrm{SD}$ ) of NR3A labeling in the presence (co-IP with $6 \mathrm{~F} 9,4.4 \pm 1.9$; co-IP with NR1, $66.9 \pm 14.8$ ) and absence (co-IP with 6F9, $60.6 \pm 8.0$; co-IP with NR1, $68.5 \pm 8.8$ ) of NMDAR stimulation (co-IP with $6 \mathrm{~F} 9, p<0.005$; co-IP with NR1, $p<$ $0.5 ; n=3$ separate experiments). $C$, Lysates from cerebrocortical neurons were separated by SDS-PAGE, and the expression of PP2A was detected by probing with antibody PR65 (recognizing the PP2A-A subunit), antibody PR55 $\alpha$ (recognizing the PP2A-B subunit), and antibody anti-PP2Ac (recognizing the PP2A-C subunit). 


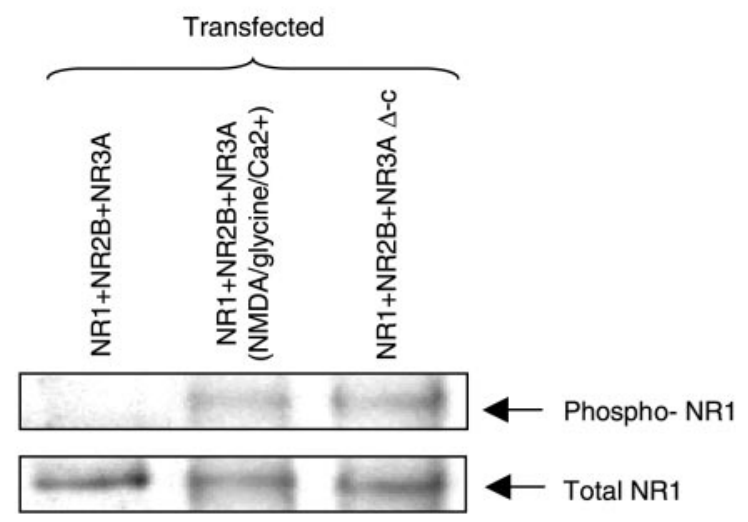

B

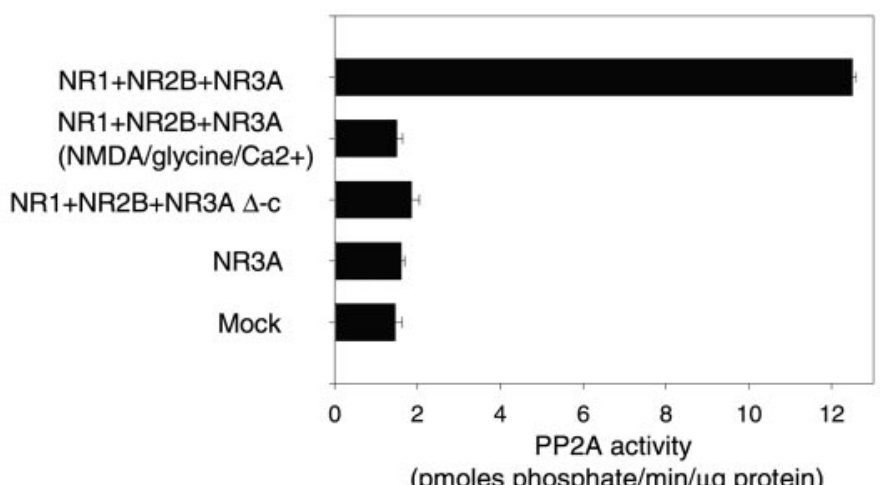

Figure 4. Dephosphorylation of the NMDAR subunit NR1 and activation of endogenous PP2A activity in transfected HEK 293 cells. A, HEK 293 cells were transiently transfected with a triple combination of NMDAR subunits consisting of NR1 + NR2B + NR3A and NR1 + $\mathrm{NR} 2 \mathrm{~B}+\mathrm{NR} 3 \mathrm{~A} \Delta$-c. After incubation in the absence or presence of NMDA $\left(200 \mu \mathrm{M}\right.$ NMDA, $10 \mu \mathrm{M}$ glycine and $2.5 \mathrm{mM} \mathrm{Ca}^{2+}$ in nominally $\mathrm{Mg}^{2+}$-free Hank's solution), cells were harvested, and lysates of transfected cells were immunoprecipitated with monoclonal antibody antiNR1. Blots were probed with monoclonal antibody anti-phospho-NR1 to detect the phosphorylated form of NR1. NR1 protein was detected by probing with monoclonal antibody anti-NR1. Three individual experiments using different batches of protein samples showed similar results. $B$, Lysates from transfected HEK 293 cells of NR1 + NR2B + NR3A, NR1 + NR2B + NR3A (treated with NMDA as above), and NR1 + NR2B + NR3A $\Delta$-c were used to assay the endogenous PP2A activity using a phosphopeptide as a substrate. Endogenous PP2A activities are given in picomoles phosphate per minute per microgram of protein \pm SD. Data are representative of three experiments showing similar results.

endogenous PP2A activity in cerebrocortical neurons (Fig. 5C) and the rodent brain at all stages of development tested (Fig. 5D).

Endogenous PP2A activity from rat brain extracts was found to peak around P8 before dropping significantly around P12 to a low level of activity in the adult (Fig. 5D). Interestingly, the developmental profile of PP2A activity appeared to parallel the protein expression profile of NR3A, which increased from $\mathrm{P} 0$ to $\mathrm{P} 8$, peaked at P8, and then decreased gradually between P12 and P20, with low levels of expression in the adult animals. In contrast, the protein expression level of NR1 showed less variation during the brain development (Fig. 5D). These data are consistent with the idea that both PP2A and NR3A are developmentally regulated in a similar way, being related to the functions of each other.

\section{DISCUSSION}

Protein phosphorylation has been established as an important mechanism for the regulation of NMDAR function. In particular, the action of protein kinases on NMDARs has been extensively studied. The NR1 subunit is a target for phosphorylation by both protein kinase A and protein kinase C (Tingley et al., 1997). For example, it was shown previously that serine residue 897 of the NR1 subunit could be phosphorylated by protein kinase A. The data of the present study indicate that the association of PP2A with the NMDAR complex that was mediated by the NR3A subunit was accompanied by the dephosphorylation of this serine residue. Interestingly, protein kinase A was reported to be closely associated with the NMDAR complex through its association with the scaffold protein yotiao (Westphal et al., 1999). In addition, another phosphatase, PP1, was also shown to be associated with yotiao. Thus, both kinases and phosphatases are located in physical proximity to their target substrates. Together, these results delineate a mechanistic framework explaining earlier findings of an electrophysiological study that demonstrated that phosphatase inhibitors enhanced NMDA currents, whereas either PP1 or PP2A decreased the open probabilities of NMDAR-gated channels (Wang et al., 1994). To date, only two features that distinguish NR3A-containing receptors from those without the subunit have been described: (1) reduced single channel conductance, and (2) reduced calcium permeability (Das et al., 1998; Pérez-Otaño et al., 2001). Both properties have been shown to be "persistent" characteristics of NR3A-containing NMDARs in the sense that neither repeated application of NMDA nor pulling of patches changed these properties. It is therefore unlikely that these biophysical properties would be influenced by PP2A or, for that matter, be dependent on the phosphorylation state of serine 897 of NR1. Thus, possible effects of the PP2A-NR3A interaction and the phosphorylation state of serine 897 of NR1 on biophysical or pharmacological properties of NMDARs remain to be established. It is conceivable, however, that the described dependence of the PP2A-NR3A interaction and the phosphorylation of serine 897 on NMDAR stimulation could be interpreted as a biochemical signal indicating the recent history of activity of individual NMDARs at synapses. Glutamatergic synapses expressing NMDA receptors containing the NR3A subunit that have not been exposed to glutamate ("unstimulated" synapses) would be characterized by dephosphorylated serine 897 of NR1. In contrast, synapses that have previously been exposed to glutamate ("stimulated" synapses) would be distinguished by phosphorylated serine 897 . Serine 897 has been shown to be directly phosphorylated by protein kinase A, which is thought to be in close proximity to NR1 through binding to the NR1 associated protein yotiao (Westphal et al., 1999). It is not known whether or not the association between yotiao and NR1 is itself regulated by phosphorylation.

Thus, it is possible to speculate that protein kinase A, PP2A, and NMDARs are part of a signaling mechanism linking the history of NMDAR stimulation to the phosphorylation of state of serine 897 of NR1. Along these lines, the finding that NR3A knock-out mice showed an increased density of spines in cortical neurons might indicate that such a signaling pathway could be involved in the regulation of the growth of dendritic spines. In fact, NMDAR activity has been implicated previously in the modulation of dendritic arbors (McAllister et al., 1996) and spines (Woolley and McEwen, 1994; Woolley et al., 1997), 
A

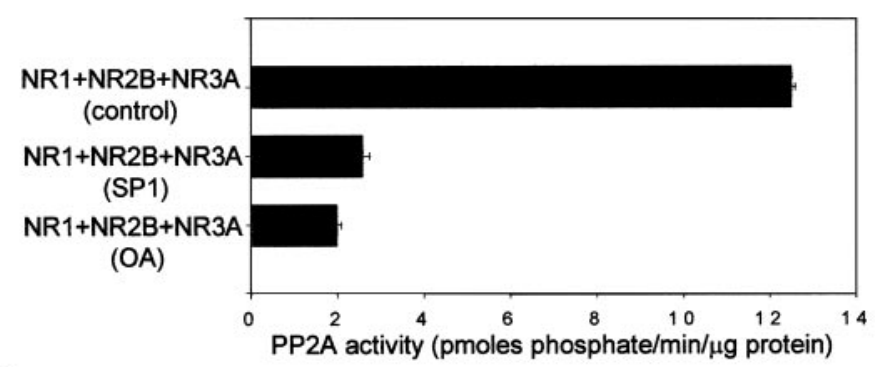

B

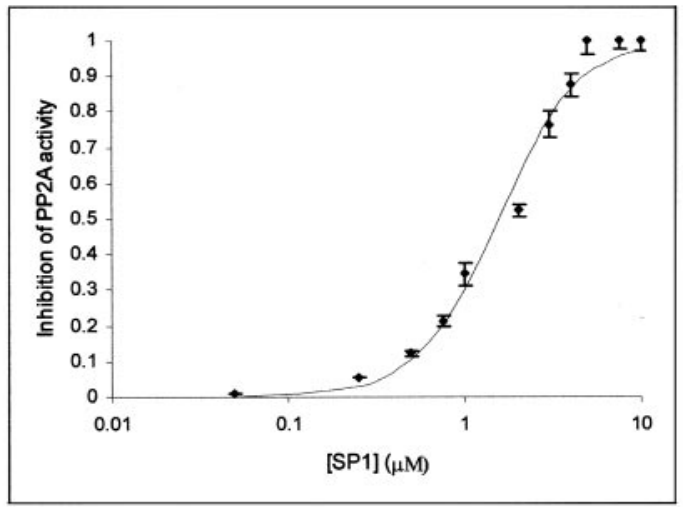

C

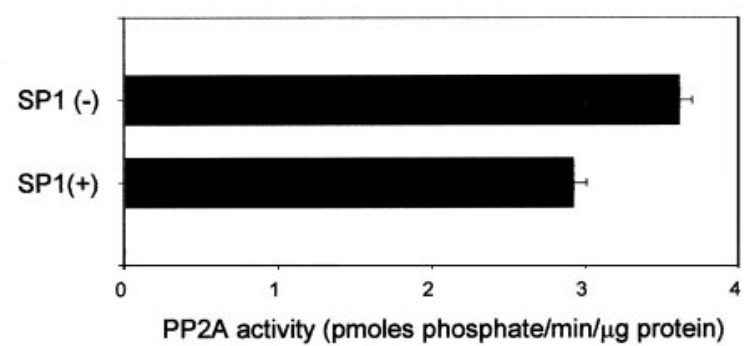

D

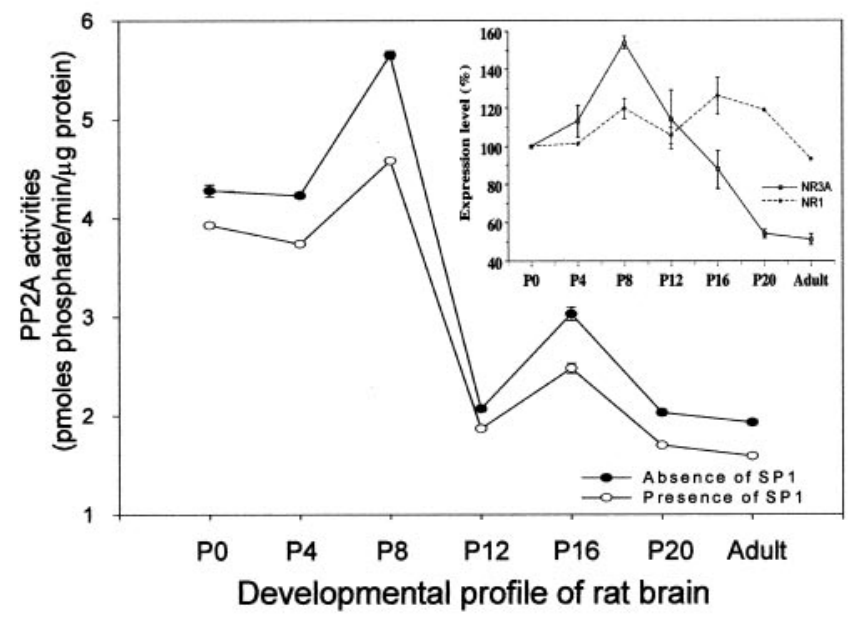

Figure 5. Blockade of endogenous PP2A activity by a synthetic peptide (SP1). A, Solubilized extract fractions from HEK 293 cells transfected with NR1 + NR2B + NR3A were used to assay the endogenous PP2A activity in the absence $\left(12.5 \pm 0.1 \mathrm{pmol} \cdot \mathrm{min}^{-1} \cdot \mu \mathrm{g}^{-1}\right)$ or presence $\left(2.6 \pm 0.2 \mathrm{pmol} \cdot \mathrm{min}^{-1} \cdot \mu \mathrm{g}{ }^{-1}\right)$ of SP1 $\left(5 \mu \mathrm{M} ; p<0.1 \times 10^{-8} ; n=3\right.$ separate experiments). Incubation with the specific PP2A inhibitor $1 \mathrm{nM}$ whereas PP2A has been shown to influence the modification of synaptic structures (Saito et al., 1995; Strack et al., 1997).

Numerous studies indicate that modification of protein phosphorylation is a critical element leading to the induction of synaptic plasticity. For example, long-term potentiation is accompanied by increased glutamate receptor phosphorylation through various protein kinases and a concomitant decrease in protein phosphatase activity (Bliss and Collingridge, 1993; Mulkey et al., 1993; Soderling and Derkach, 2000). In contrast, a decrease in synaptic strength, LTD, has been shown to be dependent on glutamate receptor dephosphorylation mediated by an increase in the activity of protein phosphatases, possibly PP1 and PP2A (Lee et al., 1998, 2000; Thiels et al., 1998). Thus, coordination of kinase and phosphatase activities is crucial for the modulation of synaptic plasticity. Intriguingly, our data demonstrate the association between NMDARs and PP2A in vivo, providing a direct link between a glutamate receptor and an associated phosphatase. In particular, the data of the present study delineate a mechanistic model of the dynamic regulation of a PP2A-NMDAR signaling complex, mediated by the interaction of NR3A and PP2A, and suggest a novel NMDAR-mediated signaling mechanism, in addition to the traditional ionotropic functions of NMDARs.

Our data indicate that the balance between kinase and phosphatase activities is subject to regulation by NMDAR activity; stimulation of NMDARs led to a decrease in the enzymatic activity of PP2A and the physical separation of PP2A from NR3A. It will be interesting to investigate the fate of the released PP2A. In particular, it will be important to characterize whether the previously NMDAR-associated PP2A serves as a signaling molecule acting on downstream targets or whether it will be recycled for binding to other, inactive NMDARs.

The ubiquitously expressed PP2A plays a major role in many cellular processes ranging from the initiation of DNA replication to cell growth and differentiation to apoptosis. The regulation of the enzymatic activity of PP2A is highly complex, involving a multitude of mechanisms (Ruediger et al., 1991; Sontag et al., 1993, 1996; Lin et al., 1998; Westphal et al., 1998; Price and Mumby, 1999). One such mechanism involves the association of the catalytic subunit of PP2A with a number of regulatory B subunits. The B subunits themselves are targets of a number of unrelated proteins that can associate with them and thus influence PP2A activity. For example, an adenovirus protein, E4orf4, has been shown to associate with PP2A through an interaction with the PP2A-B subunit, PR55 $\alpha$. The resulting PP2A-E4orf4

$\leftarrow$

OA was used as control to block endogenous PP2A activity in transfected HEK 293 cells. B, Dose-response curve for the inhibition of PP2A activity by SP1. Solubilized extract fractions from HEK 293 cells transfected with NR1 + NR2B + NR3A were used to evaluate the $\mathrm{IC}_{50}$ of SP1 on PP2A activity. The maximum inhibitory effect of SP1 was normalized to 1 . Each data point represents the average of three experiments $\pm \mathrm{SD}$. $C$, Solubilized extract fractions from cerebrocortical neurons were used to determine the endogenous PP2A activity in the absence (3.6 \pm 0.09 $\left.\mathrm{pmol} \cdot \min ^{-1} \cdot \mu \mathrm{g}^{-1}\right)$ or presence $\left(2.9 \pm 0.09 \mathrm{pmol} \cdot \min ^{-1} \cdot \mu \mathrm{g}^{-1}\right)$ of SP1 (5 $\mu \mathrm{M} ; p<0.001 ; n=3$ separate experiments). $D$, Solubilized extract fractions from rat brain at different stages of development (P0, P4, P8, P12, P16, P20, and adult) were used to assay the endogenous PP2A activity in the absence or presence of $5 \mu \mathrm{M} \mathrm{SP} 1$, and each data point represents the average of three experiments $\pm \mathrm{SD}(p<0.005$ at all points). The developmental protein expression profiles of NR3A and NR1 were determined, and the expression levels were normalized relative to $\mathrm{P} 0$ and expressed as percentage $\pm \mathrm{SD}$. Three individual experiments using different batches of protein samples showed similar results. 
complex is thought to mediate the regulation of AP-1 transcriptional activity, which occurs during the late stage of viral infection (Kleinberger and Shenk, 1993). Similarly, a complex formed by two HIV-encoded proteins, NCp7 and Vpr, has been shown to bind to and activate the PP2A holoenzyme containing the B subunit PR61 (Tung et al., 1997). Our data suggest a novel mechanism of regulation of PP2A activity through the formation of a receptor-enzyme complex involving the catalytic subunit of PP2A. Formation of such a complex might be important in a variety of signaling cascades involving PP2A.

\section{REFERENCES}

Bliss TV, Collingridge GL (1993) A synaptic model of memory: longterm potentiation in the hippocampus. Nature 361:31-39.

Ciabarra AM, Sullivan JM, Gahn LG, Pecht G, Heinemann S, Sevarino KA (1995) Cloning and characterization of $\chi-1$ : a developmentally regulated member of a novel class of the ionotropic glutamate receptor family. J Neurosci 15:6498-6508.

Das S, Sasaki YF, Rothe T, Premkumar LS, Takasu M, Crandall JE, Dikkes P, Conner DA, Rayudu PV, Cheung W, Chen HS, Lipton SA, Nakanishi N (1998) Increased NMDA current and spine density in mice lacking the NMDA receptor subunit NR3A. Nature 393:377-381.

Faux MC, Scott JD (1996) More on target with protein phosphorylation: conferring specificity by location. Trends Biochem Sci 21:312-315.

Fraser D, Scott JD (1999) Modulation of ion channels: a "current" view of AKAPs. Neuron 23:423-426.

Ho SN, Hunt HD, Horton RM, Pullen JK, Pease LR (1989) Sitedirected mutagenesis by overlap extension using the polymerase chain reaction. Gene 77:51-59.

Kennedy MB (2000) Signal-processing machines at the postsynaptic density. Science 290:750-754.

Kleinberger T, Shenk T (1993) Adenovirus E4orf4 protein binds to protein phosphatase $2 \mathrm{~A}$ and the complex down regulates E1Aenhanced junB transcription. J Virol 67:7556-7560.

Kremmer E, Ohst K, Kiefer J, Brewis N, Walter G (1997) Separation of PP2A core enzyme and holoenzyme with monoclonal antibodies against the regulatory A subunit: abundant expression of both forms in cells. Mol Cell Biol 17:1692-1701.

Kutsuwada T, Kashiwabuchi N, Mori H, Sakimura K, Kushiya E, Araki K, Meguro H, Masaki H, Kumanishi T, Arakawa M, Mishina M (1992) Molecular diversity of the NMDA receptor channel. Nature 358:36-41.

Lee HK, Kameyama K, Huganir RL, Bear MF (1998) NMDA induces long-term synaptic depression and dephosphorylation of the GluR1 subunit of AMPA receptors in hippocampus. Neuron 21:1067-1078.

Lee HK, Barbarosie M, Kameyama K, Bear MF, Huganir RL (2000) Regulation of distinct AMPA receptor phosphorylation sites during bidirectional synaptic plasticity. Nature 405:955-959.

Levitan IB (1999) Modulation of ion channels by protein phosphorylation. How the brain works. Adv Second Messenger Phosphoprotein Res 33:3-22.

Lin XH, Walter J, Scheidtmann K, Ohst K, Newport J, Walter G (1998) Protein phosphatase $2 \mathrm{~A}$ is required for the initiation of chromosomal DNA replication. Proc Natl Acad Sci USA 95:14693-14698.

McAllister AK, Katz LC, Lo DC (1996) Neurotrophin regulation of cortical dendritic growth requires activity. Neuron 17:1057-1064.

Meguro H, Mori H, Araki K, Kushiya E, Kutsuwada T, Yamazaki M, Kumanishi T, Arakawa M, Sakimura K, Mishina M (1992) Functional characterization of a heteromeric NMDA receptor channel expressed from cloned cDNAs. Nature 357:70-74.

Monyer H, Sprengel R, Schoepfer R, Herb A, Higuchi M, Lomeli H, Burnashev N, Sakmann B, Seeburg PH (1992) Heteromeric NMDA receptors: molecular and functional distinction of subtypes. Science 256:1217-1221.

Moriyoshi K, Masu M, Ishii T, Shigemoto R, Mizuno N, Nakanishi S (1991) Molecular cloning and characterization of the rat NMDA receptor. Nature 354:31-37.

Mulkey RM, Herron CE, Malenka RC (1993) An essential role for protein phosphatases in hippocampal long-term depression. Science 261:1051-1055.

Mulkey RM, Endo S, Shenolikar S, Malenka RC (1994) Involvement of a calcineurin/inhibitor-1 phosphatase cascade in hippocampal longterm depression. Nature 369:486-488.

Mumby M (1995) Regulation by tumour antigens defines a role for PP2A in signal transduction. Semin Cancer Biol 6:229-237.

Mumby MC, Walter G (1993) Protein serine/threonine phosphatases: structure regulation and functions in cell growth. Physiol Rev 73:673-699.

Pérez-Otaño I, Schulteis CT, Contractor A, Lipton SA, Trimmer JS, Sucher NJ, Heinemann SF (2001) Assembly with the NR1 subunit is required for surface expression of NR3A-containing NMDA receptors. J Neurosci 21:1228-1237.

Price NE, Mumby MC (1999) Brain protein serine/threonine phosphatases. Curr Opin Neurobiol 9:336-342.

Roche KW, Tingley WG, Huganir RL (1994) Glutamate receptor phosphorylation and synaptic plasticity. Curr Opin Neurobiol 4:383-388.

Rogers SW, Hughes TE, Hollmann M, Gasic GP, Deneris ES, Heinemann S (1991) The characterization and localization of the glutamate receptor subunit GluR1 in the rat brain. J Neurosci 11:2713-2724.

Rose MD, Winston F, Hieter P (1990) Methods in yeast genetics: a laboratory course manual. Assay of $\beta$-galactosidase in yeast, pp 155159. New York: Cold Spring Harbor Laboratory.

Ruediger R, Van Wart Hood JE, Mumby M, Walter G (1991) Constant expression and activity of protein phosphatase $2 \mathrm{~A}$ in synchronized cells. Mol Cell Biol 11:4282-4285.

Saito T, Shima H, Osawa Y, Nagao M, Hemmings BA, Kishimoto T, Hisanaga S (1995) Neurofilament-associated protein phosphatase 2A its possible role in preserving neurofilaments in filamentous states. Biochemistry 34:7376-7384.

Schonthal AH (1995) Regulation of gene expression by serine/threonine protein phosphatases. Semin Cancer Biol 6:239-248.

Sheng M, Pak DT (1999) Glutamate receptor anchoring proteins and the molecular organization of excitatory synapses. Ann NY Acad Sci 868:483-493.

Smart TG (1997) Regulation of excitatory and inhibitory neurotransmitter-gated ion channels by protein phosphorylation. Curr Opin Neurobiol $7: 358-367$.

Soderling TR, Derkach VA (2000) Postsynaptic protein phosphorylation and LTP. Trends Neurosci 23:75-80.

Sontag E, Fedorov S, Kamibayashi C, Robbins D, Cobb M, Mumby M (1993) The interaction of SV40 small tumor antigen with protein phosphatase $2 \mathrm{~A}$ stimulates the map kinase pathway and induces cell proliferation. Cell 75:887-897.

Sontag E, Nunbhakdi-Craig V, Lee G, Bloom GS, Mumby MC (1996) Regulation of the phosphorylation state and microtubule-binding activity of Tau by protein phosphatase 2A. Neuron 17:1201-1207.

Strack S, Westphal RS, Colbran RJ, Ebner FF, Wadzinski BE (1997) Protein serine/threonine phosphatase 1 and $2 \mathrm{~A}$ associate with and dephosphorylate neurofilaments. Mol Brain Res 49:15-28.

Strack S, Zaucha JA, Ebner FF, Colbran RJ, Wadzinski BE (1998) Brain protein phosphatase $2 \mathrm{~A}$ : developmental regulation and distinct cellular and subcellular localization by B subunits. J Comp Neurol 392:515-527.

Sucher NJ, Akbarian S, Chi CL, Leclerc CL, Awobuluyi M, Deitcher DL, Wu MK, Yuan JP, Jones EG, Lipton SA (1995) Developmental and regional expression pattern of a novel NMDA receptor-like subunit (NMDAR-L) in the rodent brain. J Neurosci 15:6509-6520.

Swope SL, Moss SI, Raymond LA, Huganir RL (1999) Regulation of ligand-gated ion channels by protein phosphorylation. Adv Second Messenger Phosphoprotein Res 33:49-78.

Thiels E, Norman ED, Barrionuevo G, Klann E (1998) Transient and persistent increases in protein phosphatase activity during long-term depression in the adult hippocampus in vivo. Neuroscience 86:1023-1029.

Tingley WG, Ehlers MD, Kameyama K, Doherty C, Ptak JB, Riley CT, Huganir RL (1997) Characterization of protein kinase A and protein kinase C phosphorylation of the N-methyl-D-aspartate receptor NR1 subunit using phosphorylation site-specific antibodies. J Biol Chem 272:5157-5166.

Tung HY, De Rocquigny H, Zhao LJ, Cayla X, Roques BP, Ozon R (1997) Direct activation of protein phosphatase-2A0 by HIV-1 encoded protein complex NCp7:vpr. FEBS Lett 401:197-201.

Walaas SI, Greengard P (1991) Protein phosphorylation and neuronal function. Pharmacol Rev 43:299-349.

Wang LY, Orser BA, Brautigan DL, MacDonald JF (1994) Regulation of NMDA receptors in cultured hippocampal neurons by protein phosphatases 1 and 2A. Nature 369:230-232.

Westphal RS, Anderson KA, Means AR, Wadzinski BE (1998) A signaling complex of $\mathrm{Ca}^{2+}$-calmodulin-dependent protein kinase IV and protein phosphatase 2A. Science 280:1258-1261.

Westphal RS, Tavalin SJ, Lin JW, Alto NM, Fraser ID, Langeberg LK, Sheng M, Scott JD (1999) Regulation of NMDA receptors by an associated phosphatase-kinase signaling complex. Science 285:93-96.

Woolley CS, McEwen BS (1994) Estradiol regulates hippocampal dendritic spine density via an N-methyl-D-aspartate receptor-dependent mechanism. J Neurosci 14:7680-7687.

Woolley CS, Weiland NG, McEwen BS, Schwartzkroin PA (1997) Estradiol increases the sensitivity of hippocampal CA1 pyramidal cells to NMDA receptor-mediated synaptic input: correlation with dendritic spine density. J Neurosci 17:1848-1859.

Ziff EB (1997) Enlightening the postsynaptic density. Neuron 19:11631174. 